\title{
THE DELTA STANDING WAVE SOLUTION FOR THE LINEAR SCALAR CONSERVATION LAW WITH DISCONTINUOUS COEFFICIENTS USING A SELF-SIMILAR VISCOUS REGULARIZATION
}

\author{
Xiumei Li And Chun Shen
}

\begin{abstract}
This paper is mainly concerned with the formation of delta standing wave for the scalar conservation law with a linear flux function involving discontinuous coefficients by using the self-similar viscosity vanishing method. More precisely, we use the self-similar viscosity to smooth out the discontinuous coefficient such that the existence of approximate viscous solutions to the delta standing wave for the Riemann problem is established and then the convergence to the delta standing wave solution is also obtained when the viscosity parameter tends to zero. In addition, the Riemann problem is also solved with the standard method and the instability of Riemann solutions with respect to the specific small perturbation of initial data is pointed out in some particular situations.
\end{abstract}

\section{Introduction}

In this paper, we are interested in the scalar conservation law with the linear flux function involving discontinuous coefficients as follows [14, 20]:

$$
u_{t}+(k(x) u)_{x}=0,
$$

in which $k$ and $u$ are used to denote the velocity and density in the discontinuous flow respectively. The equation (1.1) has been extensively used in many areas, such as the particle flows [2, 3, 4] and the polymer flooding of an oil reservoir [17] etc. It also arises in modeling wave propagation through interfaces where jumps in $k(x)$ correspond to interfaces between different media [20]. The equation (1.1) can also be regarded as the simplest example for hyperbolic conservation laws with discontinuous coefficients.

Received September 18, 2014; Revised March 1, 2015.

2010 Mathematics Subject Classification. 35L65, 35L67, 76N15.

Key words and phrases. linear flux function, discontinuous coefficient, delta standing wave, viscosity vanishing method, Riemann problem, scalar conservation law.

This work is supported by National Natural Science Foundation of China (11441002, 11271176) and Shandong Provincial Natural Science Foundation (ZR2014AM024). 
If $k(x)$ is allowed to be discontinuous, then the Cauchy problem for (1.1) usually does not possess a weak $L^{\infty}$-solution except for some particular initial data and thus it is natural to consider the solution $u(x, t)$ in the sense of distributions. In this situation, by using the standard technique in [19] for hyperbolic conservation laws with discontinuous coefficients, it is more convenient to view (1.1) as a system by adding the trivial term $k_{t}=0$, which enables us to alternatively consider a system

$$
\left\{\begin{array}{l}
k_{t}=0, \\
u_{t}+(k u)_{x}=0 .
\end{array}\right.
$$

In [17], Hu at first investigated the Riemann problem for the system (1.2) with the particular initial data

$$
(k, u)(x, 0)=\left(k_{ \pm}, u_{ \pm}\right), \quad \pm x>0,
$$

where $k_{ \pm}$and $u_{ \pm}$are all given constants. The Dirac measure-valued solution was introduced in the Riemann solutions in some non-classical situations and then he discovered that the Dirac measure-valued solution can be obtained as the limit of the following self-similar viscosity regularized system

$$
\left\{\begin{array}{l}
k_{t}=\varepsilon t k_{x x}, \\
u_{t}+(k u)_{x}=\varepsilon t u_{x x},
\end{array}\right.
$$

by using the standard Dafermos technique [7, 8]. In [14], Gosse and James have also obtained the Dirac measure-valued solution to the Riemann problem (1.2) and (1.3) in the numerical computation.

In this note, we consider an alternative self-similar viscosity regularized system as follows:

$$
\left\{\begin{array}{l}
k_{t}=\varepsilon t k_{x x} \\
u_{t}+(k u)_{x}=0
\end{array}\right.
$$

which enables us to see the formation of delta standing wave solution to the Riemann problem (1.2) and (1.3) in a relatively simpler manner. In other words, we only smooth out the discontinuous coefficient $k(x)$ which also enables us to establish the existence of approximate viscous solutions to the delta standing wave for the Riemann problem (1.2) and (1.3) and then obtain the convergence to the delta standing wave when the viscosity parameter tends to zero. Here we are also interested in the effect of singular diffusion matrices where the viscosity term only appears in the first equation to smooth out the discontinuous coefficient $k(x)$ and the continuity equation is unchanged.

It is well known that the delta standing wave appears in the Riemann solutions to (1.2) and (1.3) in some situations. To be more precise, for the case $k_{+}<0<k_{-}$, the Riemann solution to (1.2) and (1.3) is a delta standing wave connecting the two constant states $\left(k_{ \pm}, u_{ \pm}\right)$whose propagation speed and strength are $\sigma=0$ and $\omega(t)=\left(k_{-} u_{-}-k_{+} u_{+}\right) t$ respectively. The main purpose of this note is to describe the formation of delta standing wave solution 
to the Riemann problem (1.2) and (1.3) by using a new self-similar viscosity regularization method (1.5). Thus, we draw our attentions on the Riemann problem (1.2) and (1.3) in the situation $k_{+}<0<k_{-}$and we can see that the delta standing wave can be obtained in the limit $\varepsilon \rightarrow 0$ of the solutions to the regularized system (1.5) with the Riemann initial data (1.3). It should be emphasized that the Riemann problem (1.2) and (1.3) may have infinitely many solutions provided that the Riemann initial data (1.3) satisfy $k_{-}<0<k_{+}$ and $u_{-} u_{+}<0$. For this reason, we restrict ourselves to consider the situation $u \geq 0$ only and it is also reasonable from the physical sense due to the fact that $u$ is regarded as the flow density. In addition, we also point out that the Riemann solution to (1.2) and (1.3) may be unstable with respect to specific small perturbation of Riemann initial data (1.3) in some situations. Here we take a detailed example to illustrate this phenomenon.

In addition, the coupling of two different conservation laws at a fixed interface has been widely investigated as

$$
\left\{\begin{array}{l}
u_{t}+f_{L}(u)=0 \text { for } x<0 \\
u_{t}+f_{R}(u)=0 \text { for } x>0
\end{array}\right.
$$

where $f_{\alpha}(\alpha=L, R)$ are two smooth flux functions. The coupled Riemann problem for the system (1.6) can also be investigated provided that an interface condition is given at $x=0$ to guarantee the well-posedness of the initial value problem for the system (1.6). The self-similar viscosity regularization is also carried out in $[1,5]$ for the system (1.6) with some particular flux functions. In particular, the discontinuous linear flux function $f_{L}=k_{L} u$ and $f_{R}=k_{R} u$ was taken in [13] as the particular interest in the numerical calculation.

Recently, the formation of the delta standing wave for the scalar conservation law (1.1) has also been considered in [34] by adopting the local linearization technique to treat the discontinuous coefficient $k(x)$ in (1.1). More precisely, the linear function in the perturbed interval $[-\varepsilon, \varepsilon]$ to connect $k_{l}$ and $k_{r}$ has been adopted in order to linearize the discontinuous coefficient $k(x)$, such that the characteristics are curved in the perturbed region $(x, t) \in[-\varepsilon, \varepsilon] \times[0,+\infty)$ and the value of $u$ along each of the characteristic curves also changes in the perturbed region. In contrast to the result in [34], the Leray-type regularization technique has also been used in [28] to deal with the discontinuous coefficient $k(x)$ in (1.1), such that all the characteristics are curved in the global physical space $(x, t)$ with $t \geq 0$ and the value of $u$ along each of the characteristic curves varies with the time.

The self-similar viscosity vanishing approach was introduced by Dafermos [8] and usually known as the Dafermos regularization technique in literature. This method has been carried out widely in the construction of Riemann solutions for broad classes of $2 \times 2$ systems including the equations of isentropic, Lagrangian, gas dynamics. In particular, it has been adopted in $[22,32]$ for the admissibility of weak solutions of the Riemann problem for hyperbolic systems of conservation laws and the admissibility criterion was called as the wave-fan criterion 
in [7]. Special attentions were also paid in $[12,16,17,24,31,33,35,37,38]$ to the formation of the delta shock waves in the Riemann solutions for some hyperbolic systems of conservation laws. There exist numerous excellent papers for the related equations and results about the measure-valued solutions such as the delta shock wave for hyperbolic systems of conservation laws, see $[6,15,18,23,25,26]$ for instance.

The paper is organized as follows. In Section 2, the Riemann problem (1.2) and (1.3) is considered for the sake of self-contained. Especially, the delta standing wave does occur in the Riemann solution when $k_{+}<0<k_{-}$. In addition, the instability of Riemann solution with respect to specific small perturbation of initial data is illustrated by a detailed example. In Section 3, for the case $k_{+}<0<k_{-}$, it is shown that there exists a smooth solution for the regularized system (1.5) with the initial data (1.3). Consequently, by taking the limit $\varepsilon \rightarrow 0$, one can see that the limit of the regularized smooth solution is just the delta standing wave, which is identical with the corresponding Riemann solution to (1.2) and (1.3) when $k_{+}<0<k_{-}$.

\section{The Riemann problem for (1.2) and (1.3)}

In this section, we simply sketch some results on the Riemann problem (1.2) and (1.3) and the detailed study can be found in [17]. We can also refer to $[21,25,31,33,35,36,37]$ for the related results about the other non-strictly hyperbolic systems of conservation laws.

The characteristic eigenvalues of (1.2) are $\lambda_{1}=0$ and $\lambda_{2}=k$ respectively. Thus, it is clear to see that (1.2) is strictly hyperbolic in the phase space provided that $k \neq 0$ and is non-strictly hyperbolic on the line $k=0$. The corresponding right characteristic vectors are $\overrightarrow{r_{1}}=(k,-u)^{T}$ and $\overrightarrow{r_{2}}=(0,1)^{T}$ respectively. Thus we have $\nabla \lambda_{i} \cdot \overrightarrow{r_{i}}=0(i=1,2)$, which implies that both the two characteristic eigenvalues $\lambda_{1}$ and $\lambda_{2}$ are linearly degenerate. Therefore, the associated wave for $\lambda_{1}$ is a standing wave discontinuity denoted by $S W$ and the associated wave for $\lambda_{2}$ is a contact discontinuity denoted by $J$.

For the Riemann problem (1.2) and (1.3), if we take the self-similar transform $\xi=x / t$, then the initial value problem can be reduced to the boundary problem for the ordinary differential equations as follows:

$$
\left\{\begin{array}{l}
-\xi k_{\xi}=0 \\
-\xi u_{\xi}+(k u)_{\xi}=0
\end{array}\right.
$$

with the boundary conditions $(k, u)( \pm \infty)=\left(k_{ \pm}, u_{ \pm}\right)$.

Then for the bounded discontinuity point $\xi=\sigma$, the Rankine-Hugoniot condition holds:

$$
\left\{\begin{array}{l}
-\sigma[k]=0 \\
-\sigma[u]+[k u]=0
\end{array}\right.
$$


Therefore, we can get $\sigma_{1}=0$ and $k_{-} u_{-}=k_{+} u_{+}$which corresponds to a standing wave discontinuity and $\sigma_{2}=k_{+}=k_{-}$which corresponds to a contact discontinuity.

Now, we can construct the Riemann solutions to (1.2) and (1.3) as follows:

(1) If $k_{-}>k_{+}>0$ or $k_{+}>k_{-}>0$, then the Riemann solution to (1.2) and (1.3) can be expressed as $S W+J$ :

$$
(k, u)(x, t)= \begin{cases}\left(k_{-}, u_{-}\right), & x<0, \\ \left(k_{+}, \frac{k_{-} u_{-}}{k_{+}}\right), & 0<x<k_{+} t, \\ \left(k_{+}, u_{+}\right), & x>k_{+} t .\end{cases}
$$

(2) If $k_{+}<k_{-}<0$ or $k_{-}<k_{+}<0$, then the Riemann solution to (1.2) and (1.3) can be expressed as $J+S W$ :

$$
(k, u)(x, t)= \begin{cases}\left(k_{-}, u_{-}\right), & x<k_{-} t, \\ \left(k_{-}, \frac{k_{+} u_{+}}{k_{-}}\right), & k_{-} t<x<0, \\ \left(k_{+}, u_{+}\right), & x>0 .\end{cases}
$$

(3) If $k_{-}<0<k_{+}$, then the Riemann solution to (1.2) and (1.3) can be expressed as $J+S W+J$ :

$$
(k, u)(x, t)= \begin{cases}\left(k_{-}, u_{-}\right), & x<k_{-} t \\ \left(k_{-}, 0\right), & k_{-} t<x<0, \\ \left(k_{+}, 0\right), & 0<x<k_{+} t, \\ \left(k_{+}, u_{+}\right), & x>k_{+} t .\end{cases}
$$

Instead of the contact vacuum state in [27], here we shall construct a physically realistic solution to the Riemann problem (1.2) and (1.3) in this case.

(4) If $k_{+}<0<k_{-}$, according to the result in [17, 27], we consider that the Riemann solution to (1.2) and (1.3) is a delta standing wave discontinuity connecting the two constant states $\left(k_{-}, u_{-}\right)$and $\left(k_{+}, u_{+}\right)$. In order to deal with it, we need the following definition of a delta standing wave solution similar to the framework introduced in [9] and used in [21, 36] for the delta shock wave solution.

Definition 2.1. A two-dimensional weighted $\delta$-measure $p(s) \delta_{S}$ supported on a smooth curve which is parameterized as $S=\{(x(s), t(s)): a<s<b\}$ should be introduced to define a measure-valued solution as

$$
\left\langle p(s) \delta_{S}, \psi(x(s), t(s))\right\rangle=\int_{a}^{b} p(s) \psi(x(s), t(s)) \sqrt{x^{\prime}(s)^{2}+t^{\prime}(s)^{2}} d s
$$

for any test function $\psi \in C_{0}^{\infty}\left(R \times R_{+}\right)$.

For convenience, the parameter $s=t$ is chosen and $w(t)=\sqrt{1+\left(x^{\prime}(t)\right)^{2}} p(t)$ is used to denote the strength of the delta shock wave. Suppose that $\Gamma=$ $\left\{\gamma_{i} \mid i \in I\right\}$ is a graph in the closed upper half-plane $\{(x, t) \mid(x, t) \in(-\infty, \infty) \times$ 
$[0, \infty)\}$ which contains Lipschitz continuous arcs $\gamma_{i}$ with $i \in I$ in which $I$ is a finite index set. Also suppose that $I_{0}$ is the subset of $I$ containing all indices of arcs starting from the $x$-axis and $\Gamma_{0}=\left\{x_{j}^{0} \mid j \in I_{0}\right\}$ is the set of initial points of the $\operatorname{arcs} \gamma_{j}$ with $j \in I_{0}$.

Definition 2.2. Let $(k, u)$ be a pair of distributions where $u$ can be expressed in the form

$$
u(x, t)=\hat{u}(x, t)+\alpha(x, t) \delta(\Gamma),
$$

in which $k, \hat{u} \in L^{\infty}\left(R \times R_{+}\right)$and the singular part should be defined by

$$
\alpha(x, t) \delta(\Gamma)=\sum_{i \in I} \alpha_{i}(x, t) \delta\left(\gamma_{i}\right) .
$$

Let us consider the initial data of the form

$$
(k, u)(x, 0)=\left(k_{0}(x), \hat{u}_{0}(x)+\sum_{j \in I_{0}} \alpha_{j}\left(x_{j}^{0}, 0\right) \delta\left(x-x_{j}^{0}\right)\right),
$$

in which $k_{0}, \hat{u}_{0} \in L^{\infty}(R)$, then the above pair of distributions $(k, u)$ are called as a generalized delta standing wave solution of (1.2) with the initial data (2.9) if $k(x, t)=k(x, 0)=k_{0}(x)$ and the following integral identity

$$
\begin{aligned}
& \int_{R_{+}} \int_{R}\left(\hat{u} \psi_{t}+k \hat{u} \psi_{x}\right) d x d t+\sum_{i \in I} \int_{\gamma_{i}} \alpha_{i}(x, t) \frac{\partial \psi(x, t)}{\partial l} \\
& +\int_{R} \hat{u}_{0}(x) \psi(x, 0) d x+\sum_{j \in I_{0}} \alpha_{j}\left(x_{j}^{0}, 0\right) \psi\left(x_{j}^{0}, 0\right)=0,
\end{aligned}
$$

holds for all test functions $\psi \in C_{c}^{\infty}\left(R \times R_{+}\right)$, in which $\frac{\partial \psi(x, t)}{\partial l}$ denotes the tangential derivative of a function $\psi$ on the graph $\gamma_{i}$ and $\int_{\gamma_{i}}$ denotes the line integral along the arc $\gamma_{i}$.

With the above definition, similar to that in $[17,27]$, we can also construct the Riemann solution to (1.2) and (1.3) for the situation $k_{+}<0<k_{-}$in the following theorem.

Theorem 2.3 (see $[17,27]$ ). For the situation $k_{+}<0<k_{-}$, the Riemann problem (1.2) and (1.3) has a piecewise smooth Riemann solution of the form:

$$
(k, u)(x, t)=\left\{\begin{array}{l}
\left(k_{-}, u_{-}\right), \quad x<0, \\
(0, \omega(t) \delta(x)), \quad x=0, \\
\left(k_{+}, u_{+}\right), \quad x>0,
\end{array}\right.
$$

where the strength of delta standing wave can be calculated by

$$
\omega(t)=\left(k_{-} u_{-}-k_{+} u_{+}\right) t .
$$

It is known that certain instability properties with respect to $L^{1}$-perturbations of initial data is presented for conservation laws with discontinuous flux. This result can also be applied to some certain Riemann solutions to (1.2) and 
(1.3) for the reason that a piecewise constant approximation also generates an approximate solution with erroneous qualitative behavior. To be more precise, as in [30], we can also consider the perturbed Riemann problem for the system (1.2) with three pieces of constant states as follows:

$$
(k, u)(x, 0)= \begin{cases}\left(k_{-}, u_{-}\right), & x<-\varepsilon, \\ \left(k_{m}, u_{m}\right), & -\varepsilon<x<\varepsilon, \\ \left(k_{+}, u_{+}\right), & x>\varepsilon,\end{cases}
$$

in which the perturbation parameter $\varepsilon$ is positive.

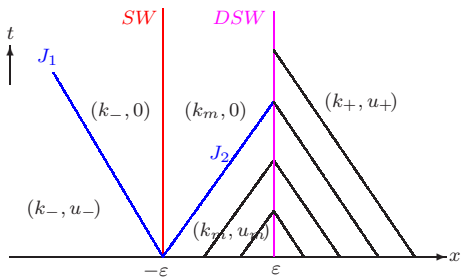

(a)

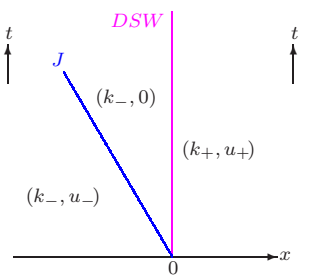

(b)

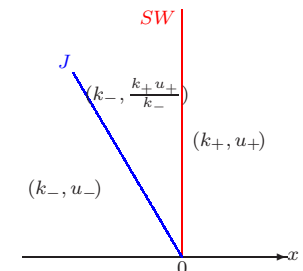

(c)

Figure 1. (a) The solution to the perturbed Riemann problem for (1.2) and (2.13) for a given positive number $\varepsilon$ when $k_{ \pm}<0<k_{m}$. (b) The limit $\varepsilon \rightarrow 0$ of the perturbed Riemann solution to (1.2) and (2.13) when $k_{ \pm}<0<k_{m}$. (c) The corresponding solution to the Riemann problem (1.2) and (1.3) when $k_{ \pm}<0$.

Let us take $k_{ \pm}<0<k_{m}$ as a detailed example to illustrate the situation that the change in the value of the discontinuous coefficient $k(x)$ in the small perturbation region $[-\varepsilon, \varepsilon]$ may change the behavior of the flow greatly. In this situation, it is clear to see that $J_{1}+S W+J_{2}$ starts from $(-\varepsilon, 0)$ and the delta standing wave denoted with $D S W$ starts from $(\varepsilon, 0)$ before the interaction happens. One can see that $J_{2}$ meets $D S W$ at $\left(x_{1}, t_{1}\right)=\left(\varepsilon, \frac{2 \varepsilon}{k_{m}}\right)$ and the strength of $D S W$ before the time $t_{1}$ can be calculated by

$$
w(t)=\left(k_{m} u_{m}-k_{+} u_{+}\right) t \quad \text { for } \quad t \leq t_{1} .
$$

At the time $t_{1}$, a new Riemann problem with delta initial data can be formulated at the intersection point $\left(x_{1}, t_{1}\right)$ whose initial data can be expressed as

$$
(k, u)\left(x, t_{1}\right)= \begin{cases}\left(k_{m}, 0\right), & x<\varepsilon, \\ \left(0, w\left(t_{1}\right) \delta(x)\right), & x=\varepsilon, \\ \left(k_{+}, u_{+}\right), & x>\varepsilon .\end{cases}
$$

The solution is still a delta standing wave on the line $x=\varepsilon$ connecting the two constant states $\left(k_{m}, 0\right)$ and $\left(k_{+}, u_{+}\right)$, whose strength can also be calculated by

$$
w(t)=\left(k_{m} u_{m}-k_{+} u_{+}\right) t_{1}-k_{+} u_{+}\left(t-t_{1}\right)=k_{m} u_{m} t_{1}-k_{+} u_{+} t \text { for } t>t_{1} \text {. }
$$


From the above discussion, the solution to the perturbed Riemann problem (1.2) and (2.13) can be constructed globally. Let us draw Figure 1(a) to illustrate it. It is clear to see that the limit $\varepsilon \rightarrow 0$ of the solution to the perturbed Riemann problem (1.2) and (2.13) is a contact discontinuity followed by a delta standing wave with the state $\left(k_{-}, 0\right)$ between them which can be described as (see Fig. 1(b)):

$$
(k, u)(x, t)= \begin{cases}\left(k_{-}, u_{-}\right), & x<k_{-} t \\ \left(k_{-}, 0\right), & k_{-} t<x<0, \\ \left(0,-k_{+} u_{+} t \delta(x)\right), & x=0, \\ \left(k_{+}, u_{+}\right), & x>0 .\end{cases}
$$

Clearly, the solution (2.17) in this limit situation is obviously different from the corresponding Riemann solution (2.4) (see Fig. 1(c)) for the case $k_{ \pm}<0$. Thus, we can see that the Riemann solution is unstable with respect to this small perturbation of Riemann initial data, which reflects the fact that the Riemann solutions to (1.2) and (1.3) are very sensitive with respect to some small perturbations of initial data.

\section{Viscous regularization of delta standing wave solution}

In this section, we draw our attentions on the viscous regularization of delta standing wave solution to the Riemann problem (1.2) and (1.3). Thus, we shall consider the Riemann problem for the viscous regularized system (1.5) with the Riemann initial data (1.3) and take one step further to restrict ourselves on the situation $k_{+}<0<k_{-}$only.

By taking the self-similar transformation $\xi=x / t$ on the viscous system (1.5) and the Riemann initial data (1.3), one can get the following boundary value problem:

$$
\left\{\begin{array}{l}
-\xi k_{\xi}=\varepsilon k_{\xi \xi}, \\
-\xi u_{\xi}+(k u)_{\xi}=0,
\end{array}\right.
$$

with the boundary conditions

$$
(k, u)( \pm \infty)=\left(k_{ \pm}, u_{ \pm}\right) .
$$

In view of the form of the system (3.1), one can see that the solution of the first equation in (3.1) does not depend on that of the second one in (3.1), such that we may only consider the first equation in (3.1) in the beginning. In other words, we may first consider

$$
\left\{\begin{array}{c}
-\xi k_{\xi}=\varepsilon k_{\xi \xi} \\
k( \pm \infty)=k_{ \pm}
\end{array}\right.
$$

For the sake of completeness, we describe the result obtained in [17] in the following lemma. 
Lemma 3.1. For each fixed $\varepsilon>0$, if $k_{+}<0<k_{-}$, then there exists a unique and monotonic smooth solution $k^{\varepsilon}(\xi)$ to the boundary value problem (3.3). To be more precise, in the case of $k_{+}<0<k_{-}, k^{\varepsilon}(\xi)$ can be expressed in a fully explicit form as

$$
k^{\varepsilon}(\xi)=k_{-}+\frac{1}{\sqrt{2 \pi \varepsilon}}\left(k_{+}-k_{-}\right) \int_{-\infty}^{\xi} \exp \left(\frac{-s^{2}}{2 \varepsilon}\right) d s .
$$

According to the expression of $k^{\varepsilon}(\xi)$ in (3.4), one can easily check the existence, smoothness, uniqueness and monotonicity of the solution to (3.3). Now, we turn our attentions on the second equation in (3.1) and consider the existence of the corresponding solution $u^{\varepsilon}(\xi)$. Based on the expression of $k^{\varepsilon}(\xi)$ in (3.4), one can obtain the following theorem to depict the corresponding solution $u^{\varepsilon}(\xi)$.

Theorem 3.2. For each fixed $\varepsilon>0$, let us suppose that $u^{\varepsilon}(\xi)$ is a weak solution of the following boundary value problem:

$$
\left\{\begin{array}{l}
-\xi u_{\xi}+\left(k^{\varepsilon} u\right)_{\xi}=0, \\
u( \pm \infty)=u_{ \pm},
\end{array}\right.
$$

where $k^{\varepsilon}(\xi)$ is given by (3.4), such that we also have $u^{\varepsilon}(\xi) \in L^{1}(-\infty,+\infty)$ which can be formulated as

$$
u^{\varepsilon}(\xi)=\left\{\begin{array}{cc}
u_{-} \exp \left(\int_{-\infty}^{\xi} \frac{k_{s}^{\varepsilon}}{s-k^{\varepsilon}} d s\right) & \text { for } \xi<\xi_{\alpha}^{\varepsilon}, \\
u_{+} \exp \left(\int_{\xi}^{+\infty} \frac{-k_{s}^{\varepsilon}}{s-k^{\varepsilon}} d s\right) & \text { for } \xi>\xi_{\alpha}^{\varepsilon},
\end{array}\right.
$$

where $\xi_{\alpha}^{\varepsilon}$ is the unique fixed point of $\xi=k^{\varepsilon}(\xi)$.

Proof. Due to the fact that $k^{\varepsilon}(\xi)$ is monotonically decreasing, the singularity point of (3.5) is unique and can be given by the solution of $\xi=k^{\varepsilon}(\xi)$ which can be denoted with $\xi_{\alpha}^{\varepsilon}$. The formula (3.6) can be derived directly from the equation in (3.5) through integrating from $-\infty$ to $\xi$ for $\xi<\xi_{\alpha}^{\varepsilon}$ and from $\xi$ to $+\infty$ for $\xi>\xi_{\alpha}^{\varepsilon}$, respectively. It is clear to see from $(3.6)$ that $u^{\varepsilon}(\xi)$ is monotonically increasing for $u_{-}>0$ or monotonically decreasing for $u_{-}<0$ in the interval $\left(-\infty, \xi_{\alpha}^{\varepsilon}\right)$, and $u^{\varepsilon}(\xi)$ is monotonically decreasing for $u_{+}>0$ or monotonically increasing for $u_{+}<0$ in the interval $\left(\xi_{\alpha}^{\varepsilon},+\infty\right)$. Furthermore, we have

$$
\begin{array}{ll}
\lim _{\xi \rightarrow \xi_{\alpha}^{\varepsilon}-} u^{\varepsilon}(\xi)= \pm \infty & \left(u_{-} \gtrless 0\right), \\
\lim _{\xi \rightarrow \xi_{\alpha}^{\varepsilon}+} u^{\varepsilon}(\xi)= \pm \infty & \left(u_{+} \gtrless 0\right) .
\end{array}
$$

Thus, the solution of (3.5) can be obtained and expressed as (3.6) by gathering the two solutions in the regions $\left(-\infty, \xi_{\alpha}^{\varepsilon}\right)$ and $\left(\xi_{\alpha}^{\varepsilon},+\infty\right)$ together. 
Hereafter, let us prove that $u^{\varepsilon}(\xi)$ is a weak solution of (3.5). Integrating the equation in $(3.5)$ on $[-R, \xi]$ for $-R<\xi<\xi_{\alpha}^{\varepsilon}$, we have

$$
\int_{-R}^{\xi} u(s) d s+u(\xi)\left(k^{\varepsilon}(\xi)-\xi\right)=u(-R)\left(k^{\varepsilon}(-R)-R\right) .
$$

Let us introduce

$$
p(\xi)=\int_{-R}^{\xi} u(s) d s, \quad a(\xi)=k^{\varepsilon}(\xi)-\xi, \quad A=u(-R)\left(k^{\varepsilon}(-R)-R\right) .
$$

Then, the equation (3.9) can be reformulated as

$$
\left\{\begin{array}{l}
a(\xi) p^{\prime}(\xi)+p(\xi)=A \\
p(-R)=0
\end{array}\right.
$$

which enables us to have

$$
p(\xi)=A\left[1-\exp \left(-\int_{-R}^{\xi} \frac{d s}{a(s)}\right)\right] .
$$

Recalling that $a(\xi)=O\left(\left|\xi-\xi_{\alpha}^{\varepsilon}\right|\right)$ as $\xi \longrightarrow \xi_{\alpha}^{\varepsilon}-0$, one can see that

$$
\lim _{\xi \rightarrow \xi_{\alpha}^{\varepsilon}-0} \int_{-R}^{\xi} \frac{d s}{a(s)}=+\infty .
$$

Passing to the limit $\xi \longrightarrow \xi_{\alpha}^{\varepsilon}-0$ in (3.11) yields

$$
\lim _{\xi \rightarrow \xi_{\alpha}^{\varepsilon}-0} \int_{-R}^{\xi} u(s) d s=\lim _{\xi \rightarrow \xi_{\alpha}^{\varepsilon}-0} p(\xi)=A .
$$

In view of $(3.9)$, it is clear to see that

$$
\lim _{\xi \rightarrow \xi_{\alpha}^{\varepsilon}-0} u(\xi)\left(k^{\varepsilon}(\xi)-\xi\right)=0 .
$$

Like as before, one can also conclude that

$$
\begin{gathered}
\lim _{\xi \rightarrow \xi_{\alpha}^{\varepsilon}+0} \int_{\xi}^{R} u(s) d s=u(R)\left(R-k^{\varepsilon}(R)\right), \\
\lim _{\xi \rightarrow \xi_{\alpha}^{\varepsilon}+0} u(\xi)\left(\xi-k^{\varepsilon}(\xi)\right)=0 .
\end{gathered}
$$

Combining the equalities (3.13) and (3.15) together implies that

$$
u^{\varepsilon}(\xi) \in L^{1}(-R,+R) \text {. }
$$

We are now in a position to prove that

$$
\left\langle-\xi u_{\xi}+\left(k^{\varepsilon} u\right)_{\xi}, \psi\right\rangle=0
$$

holds for any test function $\psi \in C_{0}^{\infty}[-R, R]$. If $\xi_{1}$ and $\xi_{2}$ satisfy $-R<\xi_{1}<$ $\xi_{\alpha}^{\varepsilon}<\xi_{2}<R$, then we have

$$
I=\int_{-R}^{R}\left(u(\xi)\left(\xi-k^{\varepsilon}(\xi)\right) \psi^{\prime}(\xi)+u(\xi) \psi(\xi)\right) d \xi
$$




$$
\begin{aligned}
& =\left\{\int_{-R}^{\xi_{1}}+\int_{\xi_{1}}^{\xi_{2}}+\int_{\xi_{2}}^{R}\right\}\left(u(\xi)\left(\xi-k^{\varepsilon}(\xi)\right) \psi^{\prime}(\xi)+u(\xi) \psi(\xi)\right) d \xi \\
& =I_{1}+I_{2}+I_{3} .
\end{aligned}
$$

It is remarkable that

$$
-\left(u(\xi)\left(\xi-k^{\varepsilon}(\xi)\right)\right)_{\xi}+u(\xi)=-\xi u_{\xi}+\left(k^{\varepsilon} u\right)_{\xi}=0 .
$$

By using the method of integration by parts, we can estimate from (3.14) and (3.16) as follows:

$$
\begin{aligned}
& \left|I_{1}\right|=\left|u\left(\xi_{1}\right)\left(\xi_{1}-k^{\varepsilon}\left(\xi_{1}\right)\right) \psi\left(\xi_{1}\right)\right| \rightarrow 0 \text { as } \xi_{1} \rightarrow \xi_{\alpha}^{\varepsilon}-, \\
& \left|I_{3}\right|=\left|u\left(\xi_{2}\right)\left(\xi_{2}-k^{\varepsilon}\left(\xi_{2}\right)\right) \psi\left(\xi_{2}\right)\right| \rightarrow 0 \text { as } \xi_{2} \rightarrow \xi_{\alpha}^{\varepsilon}+.
\end{aligned}
$$

On the other hand, for $u(\xi) \in L^{1}(-R,+R)$, we have

$$
\left|I_{2}\right| \leq \int_{\xi_{1}}^{\xi_{2}}|u(\xi)|\left(\left|\xi-k^{\varepsilon}(\xi)\right|\left|\psi^{\prime}(\xi)\right|+|\psi(\xi)|\right) d \xi \rightarrow 0
$$

as $\xi_{1} \rightarrow \xi_{\alpha}^{\varepsilon}-0$ and $\xi_{2} \rightarrow \xi_{\alpha}^{\varepsilon}+0$.

For the above discussion, we can see that $I=0$ is independent of the choices of $\xi_{1}$ and $\xi_{2}$ in the sense of distributions, which enables us to see that (3.17) is true in the weak sense. Hence, $u^{\varepsilon}(\xi)$ defined in (3.6) is the unique weak solution of (3.5) for the reason that $R$ is any sufficiently large and real number.

Let us denote $\xi_{\alpha}=\lim _{\varepsilon \rightarrow 0^{+}} \alpha^{\varepsilon}$ to express the limit of the unique fixed point $\xi_{\alpha}^{\varepsilon}$ satisfying $\xi=k^{\varepsilon}(\xi)$, then this point will play a very important role in the following discussion.

Lemma 3.3. For any $\eta>0$, we have that

$$
\lim _{\varepsilon \rightarrow 0^{+}} k^{\varepsilon}(\xi)= \begin{cases}k_{-} & \text {for } \xi<\xi_{\alpha}-\eta, \\ k_{+} & \text {for } \xi>\xi_{\alpha}+\eta,\end{cases}
$$

holds uniformly in the above intervals.

Proof. For given $\eta>0$, it is easy to take $\varepsilon>0$ sufficiently small such that the inequality $\xi_{\alpha}^{\varepsilon}<\xi_{\alpha}+\frac{\eta}{4}$ is satisfied for the reason that $\xi_{\alpha}=\lim _{\varepsilon \rightarrow 0^{+}} \xi_{\alpha}^{\varepsilon}$. If we take $\xi_{1}=\xi_{\alpha}+\frac{\eta}{2}$, then it is clear to see that $\xi_{\alpha}^{\varepsilon}<\xi_{1}-\frac{\eta}{4}$. For $k_{+}<0<k_{-}$, one achieves $k_{\xi}^{\varepsilon}(\xi)<0$. Then, it follows from (3.3) that

$$
\frac{-\varepsilon}{\xi}=\frac{k_{\xi \xi}^{\varepsilon}(\xi)}{k_{\xi}^{\varepsilon}(\xi)}
$$

By integrating (3.19) from $\xi_{1}$ to $\xi$, one can arrive at

$$
k_{\xi}^{\varepsilon}(\xi)=k_{\xi}^{\varepsilon}\left(\xi_{1}\right) \exp \left(\int_{\xi_{1}}^{\xi}-\frac{s}{\varepsilon} d s\right) .
$$

Furthermore, integrating (3.20) from $\xi_{1}$ to $+\infty$ again leads to

$$
k_{+}-k^{\varepsilon}\left(\xi_{1}\right)=k_{\xi}^{\varepsilon}\left(\xi_{1}\right) \int_{\xi_{1}}^{+\infty} \exp \left(\int_{\xi_{1}}^{\xi}-\frac{s}{\varepsilon} d s\right) d \xi .
$$


Because $k^{\varepsilon}(\xi)$ is monotonically decreasing in the real axis, we have $k_{\xi}^{\varepsilon}\left(\xi_{1}\right)<0$ and $k_{+} \leq k^{\varepsilon}(s) \leq k_{-}$. Thus, for a sufficiently small positive number $\varepsilon$, one can conclude that

$$
\begin{aligned}
k_{+}-k_{-} & \leq k_{+}-k^{\varepsilon}\left(\xi_{1}\right) \\
& =k_{\xi}^{\varepsilon}\left(\xi_{1}\right) \int_{\xi_{1}}^{+\infty} \exp \left(\int_{\xi_{1}}^{\xi}-\frac{s}{\varepsilon} d s\right) d \xi \\
& =k_{\xi}^{\varepsilon}\left(\xi_{1}\right) \int_{\xi_{1}}^{+\infty} \exp \left(-\frac{1}{2 \varepsilon}\left(\xi-\xi_{1}\right)\left(\xi+\xi_{1}\right)\right) d \xi \\
& =k_{\xi}^{\varepsilon}\left(\xi_{1}\right) \int_{0}^{+\infty} \exp \left(-\frac{1}{2 \varepsilon} \zeta\left(\zeta+2 \xi_{1}\right)\right) d \zeta \\
& \leq k_{\xi}^{\varepsilon}\left(\xi_{1}\right) \cdot \varepsilon C_{1}
\end{aligned}
$$

in which the change of variables $\zeta=\xi-\xi_{1}$ has been used and $C_{1}$ is a constant independent of $\varepsilon$. Thus, we can get $0>k_{\xi}^{\varepsilon}\left(\xi_{1}\right) \geq \frac{k_{+}-k_{-}}{\varepsilon C_{1}}$. Together with (3.20), we arrive at

$$
0>k_{\xi}^{\varepsilon}(\xi) \geq \frac{k_{+}-k_{-}}{\varepsilon C_{1}} \cdot \exp \left(\int_{\xi_{1}}^{\xi}-\frac{s}{\varepsilon} d s\right) .
$$

Hence, we know that $k_{\xi}^{\varepsilon} \rightarrow 0$ as $\varepsilon \rightarrow 0$ holds uniformly on $\xi>\xi_{\alpha}+\eta$.

For any $\xi>\xi_{\alpha}+\eta$, we have

$$
k_{+}-k^{\varepsilon}(\xi)=\int_{\xi}^{+\infty} k_{s}^{\varepsilon}(s) d s .
$$

Obviously, we can draw the conclusion that $\lim _{\varepsilon \rightarrow 0^{+}} k^{\varepsilon}(\xi)=k_{+}$holds uniformly on $\xi>\xi_{\alpha}+\eta$ in view of (3.22).

Next, let us prove that $\lim _{\varepsilon \rightarrow 0^{+}} k^{\varepsilon}(\xi)=k_{-}$holds uniformly on $\xi<\xi_{\alpha}-\eta$. Similarly, it is easy to take $\varepsilon>0$ sufficiently small such that $\xi_{\alpha}^{\varepsilon}>\xi_{\alpha}-\frac{\eta}{4}$. If we take $\xi_{3}=\xi_{\alpha}-\frac{\eta}{2}$, then we also have $\xi_{\alpha}^{\varepsilon}>\xi_{3}+\frac{\eta}{4}$. By integrating (3.3) over $\left(\xi, \xi_{3}\right)$, we have

$$
k_{\xi}^{\varepsilon}(\xi)=k_{\xi}^{\varepsilon}\left(\xi_{3}\right) \exp \left(\int_{\xi_{3}}^{\xi}-\frac{s}{\varepsilon} d s\right)
$$

Consequently, by integrating $(3.22)$ over $\left(-\infty, \xi_{3}\right)$ again, we also have

$$
k^{\varepsilon}\left(\xi_{3}\right)-k_{-}=k_{\xi}^{\varepsilon}\left(\xi_{3}\right) \cdot \int_{-\infty}^{\xi_{3}} \exp \left(\int_{\xi_{3}}^{\xi}-\frac{s}{\varepsilon} d s\right) d \xi .
$$

Thus, for a sufficiently small positive number $\varepsilon$, it yields

$$
\begin{aligned}
k_{+}-k_{-} & \leq k^{\varepsilon}\left(\xi_{3}\right)-k_{-} \\
& =k_{\xi}^{\varepsilon}\left(\xi_{3}\right) \cdot \int_{-\infty}^{\xi_{3}} \exp \left(\int_{\xi_{3}}^{\xi}-\frac{s}{\varepsilon} d s\right) d \xi
\end{aligned}
$$




$$
\begin{aligned}
& =k_{\xi}^{\varepsilon}\left(\xi_{3}\right) \int_{-\infty}^{\xi_{3}} \exp \left(-\frac{1}{2 \varepsilon}\left(\xi-\xi_{1}\right)\left(\xi+\xi_{1}\right)\right) d \xi \\
& =k_{\xi}^{\varepsilon}\left(\xi_{3}\right) \int_{-\infty}^{0} \exp \left(-\frac{1}{2 \varepsilon} \zeta\left(\zeta+2 \xi_{1}\right)\right) d \zeta \\
& \leq k_{\xi}^{\varepsilon}\left(\xi_{3}\right) \cdot \varepsilon C_{2}
\end{aligned}
$$

in which the change of variables $\zeta=\xi-\xi_{3}$ has also been used and $C_{2}$ is a constant independent of $\varepsilon$. Thus, we have $0>k_{\xi}^{\varepsilon}\left(\xi_{3}\right) \geq \frac{k_{+}-k_{-}}{\varepsilon C_{2}}$. Together with (3.24), we also know that

$$
0>k_{\xi}^{\varepsilon}(\xi) \geq \frac{k_{+}-k_{-}}{\varepsilon C_{2}} \cdot \exp \left(\int_{\xi_{3}}^{\xi}-\frac{s}{\varepsilon} d s\right) .
$$

So, we can conclude that $k_{\xi}^{\varepsilon} \rightarrow 0$ as $\varepsilon \rightarrow 0$ holds uniformly on $\xi<\xi_{\alpha}-\eta$.

Thus, for any $\xi<\xi_{\alpha}-\eta$, we have

$$
k^{\varepsilon}(\xi)-k_{-}=\int_{-\infty}^{\xi} k_{s}^{\varepsilon}(s) d s .
$$

Obviously, we can see that $\lim _{\varepsilon \rightarrow 0^{+}} k^{\varepsilon}(\xi)=k_{-}$holds uniformly on $\xi<\xi_{\alpha}-$ $\eta$.

Lemma 3.4. Let $\xi_{\alpha}$ be defined as above. Then, in the case of $k_{+}<0<k_{-}$, we have

$$
\xi_{\alpha}=0 .
$$

Proof. Take any $\phi(\xi) \in C_{0}^{\infty}\left(\xi_{1}, \xi_{2}\right)$ where $\xi_{1}<\xi_{\alpha}<\xi_{2}$. It follows from (3.3) that

$$
\int_{\xi_{1}}^{\xi_{2}} \varepsilon k_{\xi \xi} \cdot \phi(\xi) d \xi=\int_{\xi_{1}}^{\xi_{2}}-\xi k_{\xi} \cdot \phi(\xi) d \xi
$$

By using integration by parts and applying the fact that $\phi$ is compactly support in the interval $\left(\xi_{1}, \xi_{2}\right)$, we have

$$
\int_{\xi_{1}}^{\xi_{2}} \varepsilon k \phi_{\xi \xi}(\xi) d \xi=\int_{\xi_{1}}^{\xi_{2}} k\left(\xi \phi_{\xi}(\xi)+\phi(\xi)\right) d \xi .
$$

By taking the limit $\varepsilon \rightarrow 0^{+}$in (3.30), it can be obtained from Lemma 3.3 that

$$
\int_{\xi_{1}}^{\xi_{\alpha}} k_{-}\left(\xi \phi_{\xi}(\xi)+\phi(\xi)\right) d \xi+\int_{\xi_{\alpha}}^{\xi_{2}} k_{+}\left(\xi \phi_{\xi}(\xi)+\phi(\xi)\right) d \xi=0 .
$$

Simplifying (3.31) leads to

$$
\left(k_{-}-k_{+}\right) \xi_{\alpha} \phi\left(\xi_{\alpha}\right)=0,
$$

which enables us to have $\xi_{\alpha}=0$ for the arbitrary of $\phi(\xi)$.

Lemma 3.5. For arbitrary $\eta>0$, in the case of $k_{+}<0<k_{-}$, we have that

$$
\lim _{\varepsilon \rightarrow 0^{+}} u^{\varepsilon}(\xi)=\left\{\begin{array}{lll}
u_{-} & \text {for } & \xi<\xi_{\alpha}-\eta, \\
u_{+} & \text {for } & \xi>\xi_{\alpha}+\eta,
\end{array}\right.
$$

holds uniformly in the above intervals. 
Proof. Let us suppose that $u_{ \pm}>0$ without loss of generality, otherwise the conclusion can be drawn similarly. Let us take $\varepsilon>0$ sufficiently small, such that we have $\left|\xi_{\alpha}^{\varepsilon}-\xi_{\alpha}\right|<\eta / 2$. For any $\xi_{1} \leq \xi_{\alpha}-\eta$, we can also have $\xi_{1} \leq \xi_{\alpha}^{\varepsilon}-\eta / 2$ for $\varepsilon$ sufficiently small.

Integrating $(3.5)$ over $\left(-\infty, \xi_{1}\right)$ gives

$$
u^{\varepsilon}\left(\xi_{1}\right)=u_{-} \exp \left(\int_{-\infty}^{\xi_{1}} \frac{-k_{s}^{\varepsilon}(s)}{k^{\varepsilon}(s)-s} d s\right) .
$$

For $s \in\left(-\infty, \xi_{1}\right)$ and $k_{+}<k^{\varepsilon}(s)<k_{-}$, there exists $\xi_{2} \in\left(-\infty, \xi_{1}\right)$ such that

$$
\begin{aligned}
k^{\varepsilon}(s)-s & \geq k^{\varepsilon}\left(\xi_{1}\right)-\xi_{1} \\
& =k^{\varepsilon}\left(\xi_{1}\right)-\xi_{1}-k^{\varepsilon}\left(\xi_{\alpha}^{\varepsilon}\right)+\xi_{\alpha}^{\varepsilon} \\
& =\left(1-k_{s}^{\varepsilon}\left(\xi_{2}\right)\right)\left(\xi_{\alpha}^{\varepsilon}-\xi_{1}\right) \\
& \geq \eta / 2 .
\end{aligned}
$$

Thus, it can be derived from (3.34) that

$$
u^{\varepsilon}\left(\xi_{1}\right) \leq u_{-} \exp \int_{-\infty}^{\xi_{1}} \frac{-2 k_{s}^{\varepsilon}(s)}{\eta} d s=u_{-} \exp \left(\frac{2 k_{-}-2 k^{\varepsilon}\left(\xi_{1}\right)}{\eta}\right) .
$$

It can be seen from Lemma 3.3 that $\lim _{\varepsilon \rightarrow 0^{+}} k^{\varepsilon}\left(\xi_{1}\right)=k_{-}$holds uniformly for $\xi_{1}<\xi_{\alpha}-\eta$. Thus, we can see that $u^{\varepsilon}(\xi) \leq u_{-}$for any $\xi<\xi_{\alpha}-\eta$. On the other hand, it is obvious to see that $u^{\varepsilon}(\xi)$ is monotonically increasing in the interval $\left(-\infty, \xi_{\alpha}\right)$, such that we have $u^{\varepsilon}(\xi) \geq u_{-}$for any $\xi<\xi_{\alpha}-\eta$. Therefore, we can conclude that $\lim _{\varepsilon \rightarrow 0^{+}} u^{\varepsilon}(\xi)=u_{-}$holds uniformly for $\xi<\xi_{\alpha}-\eta$.

Finally, for any $\xi_{3} \geq \xi_{\alpha}+\eta$, it is easy to see that $\xi_{3} \geq \xi_{\alpha}^{\varepsilon}+\eta / 2$ holds for sufficiently small $\varepsilon>0$. Integrating (3.5) over $\left(\xi_{3},+\infty\right)$, we have

$$
u^{\varepsilon}\left(\xi_{3}\right)=u_{+} \exp \left(\int_{\xi_{3}}^{+\infty} \frac{-k_{s}^{\varepsilon}(s)}{s-k^{\varepsilon}(s)} d s\right) .
$$

Similarly, we also have $s-k^{\varepsilon}(s) \geq \eta / 2$ for $s \in\left(\xi_{3},+\infty\right)$. Thus, we have

$$
u^{\varepsilon}\left(\xi_{3}\right) \leq u_{+} \exp \int_{\xi_{3}}^{+\infty} \frac{-2 k_{s}^{\varepsilon}(s)}{\eta} d s=u_{+} \exp \left(\frac{2 k^{\varepsilon}\left(\xi_{3}\right)-2 k_{+}}{\eta}\right) .
$$

Obviously, we can conclude that $\lim _{\varepsilon \rightarrow 0^{+}} k^{\varepsilon}\left(\xi_{3}\right)=k_{+}$holds uniformly for $\xi_{3} \geq$ $\xi_{\alpha}+\eta$. Thus, it yields $u^{\varepsilon}(\xi) \leq u_{+}$for any $\xi \geq \xi_{\alpha}+\eta$. On the other hand, $u^{\varepsilon}(\xi)$ is monotonically increasing in the interval $\left(\xi_{\alpha},+\infty\right)$, such that we have $u^{\varepsilon}(\xi) \geq u_{+}$for any $\xi \geq \xi_{\alpha}+\eta$. Therefore, the conclusion can be drawn that $\lim _{\varepsilon \rightarrow 0^{+}} u^{\varepsilon}(\xi)=u_{+}$holds uniformly for $\xi \geq \xi_{\alpha}+\eta$.

Theorem 3.6. Let $\left(k^{\varepsilon}(\xi), u^{\varepsilon}(\xi)\right)$ be the solution of (3.1) and (3.2) for $k_{+}<$ $0<k_{-}$and given $\varepsilon>0$. Then the limit of $\left(k^{\varepsilon}(\xi), u^{\varepsilon}(\xi)\right)$ as $\varepsilon \rightarrow 0^{+}$can be expressed as (2.11) with (2.12) exactly, which is identical with the corresponding Riemann solution of (1.2) and (1.3). 
Proof. Let us first investigate the limit $\varepsilon \rightarrow 0$ behavior of $u^{\varepsilon}(\xi)$ in the neighborhood of $\xi=0$. We take $\phi(\xi) \in C_{0}^{\infty}\left(\xi_{1}, \xi_{2}\right)$ with $\xi_{1}<0<\xi_{2}$ which should satisfy $\phi(\xi)=\phi(0)$ in the neighborhood of $\xi=0$ such as $N(\mu)=[-\mu, \mu]$ for sufficiently small $\mu>0$. We remark that it is called as the sloping test function in [35].

It follows from (3.5) that

$$
\int_{\xi_{1}}^{\xi_{2}}\left(-\xi u_{\xi}^{\varepsilon}+\left(k^{\varepsilon} u^{\varepsilon}\right)_{\xi}\right) \phi(\xi) d \xi=\int_{\xi_{1}}^{\xi_{2}} u^{\varepsilon}\left(\xi-k^{\varepsilon}\right) \phi_{\xi}(\xi) d \xi+\int_{\xi_{1}}^{\xi_{2}} u^{\varepsilon}(\xi) \phi(\xi) d \xi=0 .
$$

Remembering that $\phi(\xi)=\phi(0)$ when $\xi \in N(\mu)$, we have

$$
\begin{aligned}
& \lim _{\varepsilon \rightarrow 0^{+}} \int_{\xi_{1}}^{\xi_{2}} u^{\varepsilon}\left(\xi-k^{\varepsilon}\right) \phi_{\xi}(\xi) d \xi \\
= & \int_{\xi_{1}}^{-\mu} u_{-}\left(\xi-k_{-}\right) \phi_{\xi}(\xi) d \xi+\int_{\mu}^{\xi_{2}} u_{+}\left(\xi-k_{+}\right) \phi_{\xi}(\xi) d \xi \\
= & u_{-}\left(-\mu-k_{-}\right) \phi(-\mu)-u_{-} \int_{\xi_{1}}^{-\mu} \phi(\xi) d \xi-u_{+}\left(\mu-k_{+}\right) \phi(\mu)-u_{+} \int_{\mu}^{\xi_{2}} \phi(\xi) d \xi .
\end{aligned}
$$

By taking the limit $\mu \rightarrow 0$ above, we have

$$
\lim _{\varepsilon \rightarrow 0^{+}} \int_{\xi_{1}}^{\xi_{2}} u^{\varepsilon}\left(\xi-k^{\varepsilon}\right) \phi_{\xi}(\xi) d \xi=[u k] \phi(0)-\int_{\xi_{1}}^{\xi_{2}}\left(u_{-} H(-\xi)+u_{+} H(\xi)\right) \phi(\xi) d \xi,
$$

in which $H$ denotes the Heaviside function.

It can be obtained by combining (3.38) and (3.39) together that

$$
\lim _{\varepsilon \rightarrow 0^{+}} \int_{\xi_{1}}^{\xi_{2}}\left(u^{\varepsilon}(\xi)-u_{-} H(-\xi)-u_{+} H(\xi)\right) \phi(\xi) d \xi=-[u k] \phi(0)
$$

for all sloping test functions $\phi(\xi) \in C_{0}^{\infty}\left[\xi_{1}, \xi_{2}\right]$. Consequently, by taking the limits $\xi_{1} \rightarrow 0^{-}$and $\xi_{2} \rightarrow 0^{+}$in (3.40) at the same time, we can obtain that

$$
u(\xi)=\lim _{\varepsilon \rightarrow 0^{+}} u^{\varepsilon}(\xi)=u_{-} H(-\xi)+u_{+} H(\xi)-[u k] \delta(\xi) .
$$

Let us denote $u_{0}(\xi)=u_{-} H(-\xi)+u_{+} H(\xi)$ and then consider the limit of $u^{\varepsilon}(x, t)$. We need to look for the solution depending on the time $t$ due to the fact that it is no longer self-similar in the limit situation. Let $\psi(x, t)=\phi\left(\frac{x}{t}\right) \in$ $C_{0}^{\infty}\left(R \times R^{+}\right)$, then we have

$$
\lim _{\varepsilon \rightarrow 0} \int_{0}^{\infty} \int_{-\infty}^{\infty} u^{\varepsilon}\left(\frac{x}{t}\right) \psi(x, t) d x d t=\lim _{\varepsilon \rightarrow 0} \int_{0}^{\infty} t\left(\int_{-\infty}^{\infty} u^{\varepsilon}(\xi) \psi(\xi t, t) d \xi\right) d t
$$

If $t$ is taken as a parameter, then it follows from (3.40) and (3.42) that

$$
\lim _{\varepsilon \rightarrow 0} \int_{-\infty}^{\infty} u^{\varepsilon}(\xi) \psi(\xi t, t) d \xi=\int_{-\infty}^{\infty} u_{0}(\xi) \psi(\xi t, t) d \xi+\left(k_{-} u_{-}-k_{+} u_{+}\right) \psi(0, t) .
$$


By substituting $\xi=\frac{x}{t}$ into (3.43) and noticing the fact that $u_{0}\left(\frac{x}{t}\right)=u_{0}(x)$, we may rewrite $(3.43)$ as

$$
\lim _{\varepsilon \rightarrow 0} \int_{0}^{\infty} \int_{-\infty}^{\infty}\left(u^{\varepsilon}\left(\frac{x}{t}\right)-u_{0}(x)\right) \psi(x, t) d x d t=\int_{0}^{\infty}\left(k_{-} u_{-}-k_{+} u_{+}\right) t \psi(0, t) d t,
$$

which implies that the limit $\varepsilon \rightarrow 0$ of $u^{\varepsilon}$ is identical with the corresponding Riemann solution (2.11) with (2.12) for $u$ exactly when $k_{+}<0<k_{-}$.

In the end, let us consider the value of $k(\xi)$ at the discontinuity point $\xi=0$. It is clear to see that (3.5) can be reformulated into the following form

$$
\int_{\xi_{1}}^{\xi_{2}} u\left(\phi+\xi \phi_{\xi}-k \phi_{\xi}\right) d \xi=0
$$

By substituting (3.41) into (3.45), we can conclude that

$$
\int_{\xi_{1}}^{\xi_{2}}\left(u_{-} H(-\xi)+u_{+} H(\xi)-[u k] \delta(\xi)\right)\left(\phi+\xi \phi_{\xi}-k \phi_{\xi}\right) d \xi=0
$$

which enables us to have

$0=\int_{\xi_{1}}^{0^{-}}\left(\phi+\xi \phi_{\xi}-k \phi_{\xi}\right) d \xi+\int_{0^{+}}^{\xi_{2}}\left(\phi+\xi \phi_{\xi}-k \phi_{\xi}\right) d \xi+[u k]\left(\phi(0)-k(0) \phi_{\xi}(0)\right)$.

Thus, we have

$$
\begin{aligned}
& k_{-} u_{-} \phi(0)-k_{+} u_{+} \phi(0)+[u k]\left(\phi(0)-k(0) \phi_{\xi}(0)\right) \\
= & \left(k_{-} u_{-} \phi(0)-k_{+} u_{+}\right) k(0) \phi_{\xi}(0)=0,
\end{aligned}
$$

which enables us to obtain $k(0)=0$ for the arbitrariness of $\phi$.

\section{References}

[1] A. Ambroso, B. Boutin, F. Coquel, E. Godlewski, and P. G. LeFloch, Coupling two scalar conservation laws via Dafermos self-similar regularization, Numerical Mathematics and Advanced Applications 2008 (2008), 209-216.

[2] S. Bernard, J. F. Colombeau, A. Meril, and L. Remaki, Conservation laws with discontinuous coefficients, J. Math. Anal. Appl. 258 (2001), no. 1, 63-86.

[3] F. Bouchut and G. Crippa, Uniqueness, renormalization and smooth approximations for linear transport equations, SIAM J. Math. Anal. 38 (2006), no. 4, 1316-1328.

[4] F. Bouchut and F. James, One-dimensional transport equations with discontinuous coefficients, Nonlinear Anal. 32 (1998), no. 7, 891-933.

[5] B. Boutin, F. Coquel, and E. Godlewski, Dafermos regularization for interface coupling of conservation laws, Hyperbolic problems: theory, numerics, applications, 567-575, Springer, Berlin, 2008

[6] G. Q. Chen and H. Liu, Formation of $\delta$-shocks and vacuum states in the vanishing pressure limit of solutions to the Euler equations for isentropic fluids, SIAM J. Math. Anal. 34 (2003), no. 4, 925-938.

[7] C. M. Dafermos, Solutions of the Riemann problem for a class hyperbolic system of conservation laws by the viscosity method, Arch. Rational Mech. Anal. 52 (1973), 1-9.

[8] _ Admissible wave fans in nonlinear hyperbolic systems, Arch. Rational Mech. Anal. 106 (1989), no. 3, 243-260. 
[9] V. G. Danilov and D. Mitrovic, Delta shock wave formation in the case of triangular hyperbolic system of conservation laws, J. Differential Equations 245 (2008), no. 12, 3704-3734.

[10] V. G. Danilov and V. M. Shelkovich, Dynamics of propagation and interaction of $\delta$ shock waves in conservation law systems, J. Differential Equations 221 (2005), no. 2, $333-381$.

[11] _ Delta-shock waves type solution of hyperbolic systems of conservation laws, Quart. Appl. Math. 63 (2005), no. 3, 401-427.

[12] G. Ercole, Delta-shock waves as self-similar viscosity limits, Quart. Appl. Math. 58 (2000), no. 1, 177-199.

[13] E. Godlewski and P. A. Raviart, The numerical interface coupling of nonlinear hyperbolic systems of conservation laws: The scalar case, Numer. Math. 97 (2004), no. 1, 81-130.

[14] L. Gosse and F. James, Numerical approximations of one-dimensional linear conservation equations with discontinuous coefficients, Math. Comp. 69 (2000), no. 231, 9871015.

[15] L. Guo, W. Sheng, and T. Zhang, The two-dimensional Riemann problem for isentropic Chaplygin gas dynamic system, Commun. Pure Appl. Anal. 9 (2010), no. 2, 431-458.

[16] J. Hu, A limiting viscosity approach to Riemann solutions containing Delta-shock waves for non-strictly hyperbolic conservation laws, Quart. Appl. Math. 55 (1997), no. 2, 361372 .

[17] _ The Riemann problem for a resonant nonlinear system of conservation laws with Dirac-measure solutions, Proc. Roy. Soc. Edinburgh Sect. A 128 (1998), no. 1, 81-94.

[18] F. Huang and Z. Wang, Well-posedness for pressureless flow, Comm. Math. Phys. 222 (2001), no. 1, 117-146.

[19] E. Isaacson and B. Temple, Nonlinear resonance in systems of conservation laws, SIAM J. Appl. Math. 52 (1992), no. 5, 1260-1278.

[20] S. Jin and P. Qi, $l^{1}$-error estimates on the immersed interface upwind scheme for linear convertion equations with piecewise constant coefficients: a simple proof, Science China: Math. 56 (2013), 2773-2782.

[21] H. Kalisch and D. Mitrovic, Singular solutions of a fully nonlinear $2 \times 2$ system of conservation laws, Proc. Edinb. Math. Soc. (2) 55 (2012), no. 3, 711-729.

[22] Y. J. Kim, A self-similar viscosity approach for the Riemann problem in isentropic gas dynamics and the structure of the solutions, Quart. Appl. Math. 59 (2001), no. 4, $637-665$.

[23] G. Lai, W. Sheng, and Y. Zheng, Simple waves and pressure delta waves for a Chaplygin gas in multi-dimensions, Discrete Contin. Dyn. Syst. 31 (2011), no. 2, 489-523.

[24] J. Li and H. Yang, Delta-shocks as limits of vanishing viscosity for multidimensional zero-presure gas dynamics, Quart. Appl. Math. 59 (2001), no. 2, 315-342.

[25] M. Nedeljkov, Shadow waves: entropies and interactions for delta and singular shocks, Arch. Ration. Mech. Anal. 197 (2010), no. 2, 487-537.

[26] E. Yu. Panov and V. M. Shelkovich, $\delta^{\prime}$-shock waves as a new type of solutions to system of conservation laws, J. Differential Equations 228 (2006), no. 1, 49-86.

[27] C. Shen, Structural stability of solutions to the Riemann problem for a scalar conservation law, J. Math. Anal. Appl. 389 (2012), no. 2, 1105-1116.

[28] - On a regularization of a scalar conservation law with discontinuous coefficients, J. Math. Phys. 55 (2014), no. 3, 031502, 15 pp.

[29] C. Shen and M. Sun, Formation of delta shocks and vacuum states in the vanishing pressure limit of Riemann solutions to the perturbed Aw-Rascle model, J. Differential Equations 249 (2010), no. 12, 3024-3051. 
[30] _ Instability of Riemann solutions to a scalar conservation law with discontinuous flux, Z. Angew. Math. Phys. 66 (2015), no. 3, 499-515.

[31] W. Sheng and T. Zhang, The Riemann problem for the transportation equations in gas dynamics, Mem. Amer. Math. Soc. 137 (1999), no. 654, viii+77 pp.

[32] M. Slemrod and A. E. Tzavaras, A limiting viscosity approach for the Riemann problem in isentropic gas dynamics, Indiana Univ. Math. J. 38 (1989), no. 4, 1047-1074.

[33] M. Sun, Delta shock waves for the chromatography equations as self-similar viscosity limits, Quart. Appl. Math. 69 (2011), no. 3, 425-443.

[34] _ Formation of delta standing wave for a scalar conservation law with a linear flux function involving discontinuous coefficients, J. Nonlinear Math. Phys. 20 (2013), no. 2, 229-244.

[35] D. Tan, T. Zhang, and Y. Zheng, Delta-shock waves as limits of vanishing viscosity for hyperbolic systems of conservation laws, J. Differential Equations 112 (1994), no. 1, $1-32$.

[36] G. Wang, One-dimensional non-linear chromatography system and $\delta$-shock waves, Z. Angew. Math. Phys. 64 (2013), no. 5, 1451-1469.

[37] H. Yang and Y. Zhang, New developments of delta shock waves and its applications in systems of conservation laws, J. Differential Equations 252 (2012), no. 11, 5951-5993.

[38] G. Yin and K. Song, Vanishing pressure limits of Riemann solutions to the isentropic relativistic Euler system for Chaplygin gas, J. Math. Anal. Appl. 411 (2014), no. 2, $506-521$.

XiUmei Li

School of Mathematics and Statistics Science

LUDONG UNIVERSITY

Yantai, Shandong Province, 264025, P. R. China

E-mail address: 240406466@99.com

Chun Shen

School of Mathematics and Statistics Science

LUDONG UNIVERSITY

Yantai, Shandong Province, 264025, P. R. China

E-mail address: shenchun3641@sina.com 